\title{
Stability of Tokamaks with Elongated Cross Section
}

\author{
C. H. An \\ Glenn Bateman
}




\section{DISCLAIMER}

This report was prepared as an account of work sponsored by an agency of the United States Government. Neither the United States Government nor any agency Thereof, nor any of their employees, makes any warranty, express or implied, or assumes any legal liability or responsibility for the accuracy, completeness, or usefulness of any information, apparatus, product, or process disclosed, or represents that its use would not infringe privately owned rights. Reference herein to any specific commercial product, process, or service by trade name, trademark, manufacturer, or otherwise does not necessarily constitute or imply its endorsement, recommendation, or favoring by the United States Government or any agency thereof. The views and opinions of authors expressed herein do not necessarily state or reflect those of the United States Government or any agency thereof. 


\section{DISCLAIMER}

Portions of this document may be illegible in electronic image products. Images are produced from the best available original document. 


\begin{tabular}{|c|}
\hline Printed in the United States of America. Available from \\
National Technical Intormation Service \\
U.S. Department of Commerce \\
5285 Port Royal Road, Springfield, Virginia 22161 \\
Price: Printed Copy $\$ 5.25$; Microfiche $\$ 3.00$
\end{tabular}

This report was prepared as an account of work sponsored by an agency of the United States Government. Neither the United States Government nor any agency thereof, nor any of their employees, contractors, subcontractors, or their employees, makes any warranty, express or implied, nor assumes any legal liability or responsibility for any third party's use or the results of such use of any information, apparatus, product or process disclosed in this report, nor represents that its use by such third party would not-infringe privately owned rights. 
ORNL/TM-6419

Dist. Category UC-20 g

Contract No. W-7405-eng-26

FUSION ENERGY DIVISION

STABILITY OF TOKAMAKS WITH ELONGATED CROSS SECTION

C. H. An

The University of Tennessee

Knoxville, Tennessee 37916

and

Glenn Bateman

Date Published - August 1978

Prepared by the

This report was prepared as an account of work account of work United States nor the United States Department of Energy, nor any of their employees, nor any of their Energy. nor any of their employees, hor any or their contraction, subcon liabiliy or cesponsibity for the acarncy, completeres or use iness of any information apparats, product or or use funess of any in forma process disclosed, or iepreshes the ins use would nor infringe privately owned rights.

DAK RTIDGE NATIONAL LABORATORY

Oak Ridge, Tennessee 37830

operated by

UNION CARBIDE CORPORATION

for the

DEPARTMENT OF ENERGY 


\section{THIS PAGE WAS INTENTIONALLY LEFT BLANK}


CONTENTS

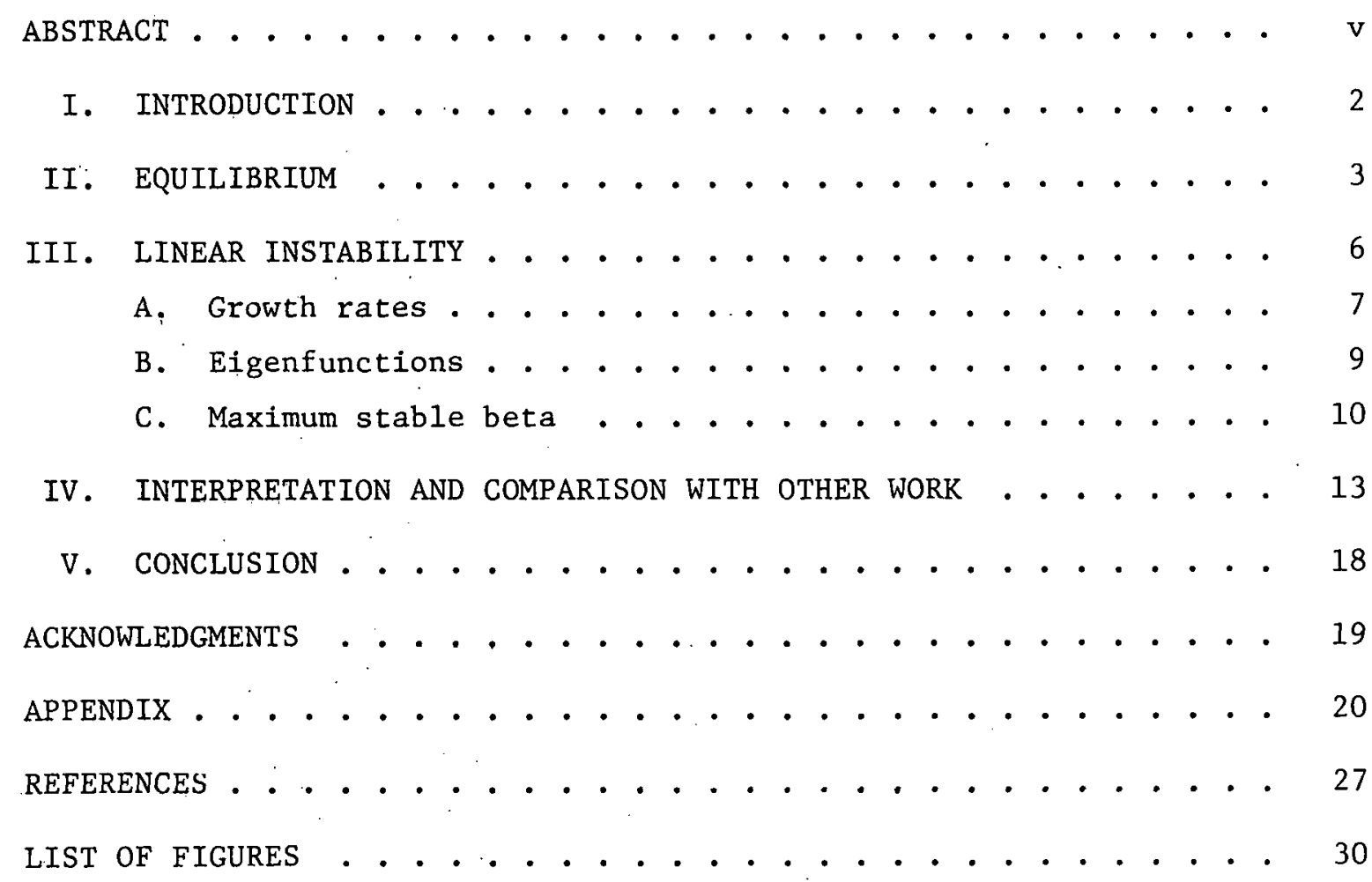


THIS PAGE

\section{WAS INTENTIONALLY \\ LEFT BLANK}




\section{ABSTRACT}

Fixed boundary $n=1$ MHD instabilities are studied computationally as a function of diamagnetism $\left(\beta_{\text {pol }}\right)$ and current profile in elongated toroidal equilibria $(1<\mathrm{b} / \mathrm{a} \leqslant 4)$. It is found that even slightly diamagnetic plasmas with broad current profile and a highly elongated cross section are subject to a ballooning instability for q-values well above unity at the magnetic axis. A peaked current profile in a mildly diamagnetic plasma decreases the elongation of the inner flux surfaces and reduces the marginal q-value by suppressing ballooning modes. The maximum stable volume-averaged beta is achleved with a broad current profile and either a paramagnetic plasma $\left(\beta_{\text {pol }}<1\right)$ with a highly elongated cross section (b/a $>2)$ or a diamagnetic plasma $\left(\beta_{\text {pol. }}>1\right)$ with only a mildly elongated cross section $(b / a<2)$. 


\section{STABILITY OF TOKAMAKS WITH ELONGATED CROSS SECTION}

C. H. An

The University of Tennessee

Knoxville, Tennessee 39916

and

Glenn Bateman

Oak Ridge National Laboratory

Oak Ridge, Tennessee 37830

\section{ABSTRACT}

Fixed boundary $\mathrm{n}=1$ MHD instabilities are studied computationally as a function of diamagnetism $\left(\beta_{\text {pol }}\right)$ and current profile in elongated toroidal equilibria $(1<\mathrm{b} / \mathrm{a} \leqslant 4)$. It is found that even slightly diamagnetic plasmas with broad current profile and a highly elongated cross section are subject to a ballooning instability for q-values well above unity at the magnetic axis. A peaked current profile in a mildly diamagnetic plasma decreases the elongation of the inner flux surfaces and reduces the marginal q-value by suppressing bailooning modes. The maximum stable volume-averaged beta is achieved with a broad current profile and elthel a paramagnetic plasma $\left(\beta_{\text {pol }}<1\right)$ with a highly elongated cross section $(\mathrm{b} / \mathrm{a}>2)$ or a diamagnetic plasma $\left(\beta_{\mathrm{pol}}>1\right)$ with only a mildly elongated cross $\operatorname{section}(b / a<2)$. 


\section{INTRODUCTION}

Elongation of the plasma cross section in tokamaks has been proposed $^{1,2}$ as a way of increasing the average beta of a plasma without violating the Kruskal-Shafranov stability criterion near the magnet1c axis, $q$-axis $\geqslant 1$. The analysis of Lortz and Nuhrenberg ${ }^{3}$ indicates that by properly shaping the plasma cross section it io also pusslble to make the plasma arbitrarily elongated without violating ihe Murcler necessury" or Lortz-sufficient ${ }^{5}$ stability criteria in the neighborhood of the magnetic axis. However, Freidberg, Haas, Marder, Goedbloed, and Grossmann ${ }^{6-9}$ have predicted that the pressure in a toroidal plasma should be limited by the onset of ballooning instabilities driven by the plasma pressure gradient in regions of convex curvature. Using a surface current model for toroidal plasmas with a variety of specified cross sectional shapes, they find that sufficiently elongated equilibria are unstable at all q-values, and that the optimum shape for maximizing beta (the plasma pressure relative to magnetic pressure) is a mildly elongated cross section with rounded ends. This optimum elongation and maximum beta depends on the details of the plasma cross sectional shape and can be slgnificantly altered by driving force-free currents outside the high pressure plagmn. ${ }^{9}$ A number of other computational and analytic predictions ${ }^{10-12}$ for highty elongated cioss sections with diffuse current profile indlcate that there may be windows of stability and enhanced beta depending on the current profile, plasma shape, and proximity of the conducting wall.

Our paper is concerned with the effects of diamagnetism and current profile on the internal MHD stability of highly elongated $(2 \leqslant \mathrm{~b} / \mathrm{a} \leqslant 4)$ toroidal plasmas with aspect ratio $\mathrm{R} / \mathrm{a}=4$ within perfectly conducting 
rectangular walls. It is found that diamagnetism and the breadth of the current profile have a strong effect on the equilibrium and stability of the plasma, and that these effects are closely interrelated. A highly peaked current profile, for example, makes the inner flux surfaces more nearly circular relative to the elongation of the walls. The geometric properties of the equilibrium have a profound effect on the overall stability of the plasma. A comparative study will be presented here to demonstrate the nature of these effects and their sensitivity.

The properties of MHD equilibria with elongated cross sections are reviewed in Sec. II. A computational study of fixed-boundary linear MHD instabilities is presented in Sec. III. We compare our results with those of other computational and analytic studies in Sec. IV. The numerical. methods used to determine the q-value at the magnetic axis and to rescale the equilibrium are presented in the Appendix.

II. ' EQUILIBRIUM

Axisymmetric toroidal equllibria with elongated rectangular boundaries are computed using a Buneman Last Poisson inverter ${ }^{3}$ to solve the GradShafranov equation 14,15

$$
\begin{aligned}
& \mathrm{RJ}_{\phi}=-\nabla * \psi=\mathrm{R}^{2} \mathrm{p}^{\prime}(\psi)+\mathrm{II}^{\prime}(\psi) \\
& \nabla^{*} \psi=\mathrm{R} \frac{\partial}{\partial \mathrm{R}} \frac{1}{\mathrm{R}} \frac{\partial \psi}{\partial \mathrm{R}}+\frac{\partial^{2} \psi}{\partial \mathrm{y}^{2}} \\
& \underset{\sim}{\mathrm{B}}=\nabla \times(\psi \nabla \phi)+\mathrm{B}_{\phi} \hat{\phi}
\end{aligned}
$$


for the stream function $\psi$ which is proportional to the poloidal flux $\psi=\psi_{\text {pol }} / 2 \pi$. The following forms are used throughout this paper for the source functions in the Grad-Shafranov equation

$$
\begin{aligned}
& p^{\prime}(\psi)=A\left(\psi / \psi_{\text {max }}\right)^{N} \\
& I(\psi)=1+C\left(\psi / \psi_{\text {max }}\right)^{M}
\end{aligned}
$$

where $\mathrm{p}(\psi)$ is the plasma pressure and $\mathrm{I}(\psi) \equiv \mathrm{B}_{\mathrm{B}} \mathrm{B}_{\phi}$ is proportional to the poloidal current $I(\psi)=I_{\text {pol }} / 2 \pi$. The stream function $\psi$ is taken to be zero on the rectangular boundary and the examples are chosen so that $\psi$ is positlve everywhere within the domain and assumes its maximum value $\psi_{\max }$ at the magnetic axis. The stream function is normalized in the source Functions in Eqs. (4) and (5) in order to improve the numerical convergence as the Grad-Shafranov equation is solved iteratively. For the studies to be presented here, the aspect ratio of the toroid was fixed at four, and grid sizes $35 \times 35$ and $35 \times 67$ (including ghost pulnts ourside the domain) were used for studying instabilities. Examples of these equilibria are illustrated in Fig. 1 and will be described in more detail below.

The coefficient C in Eq. (4) determines the paramagnetism (C >0) or diamagnetism $(C<0)$ of the plasma while the powers $N$ and $M$ control the width of the pressure profile and poloidal current profile. As the plasma is made more diamagnetic, by making the coefficient $C$ more negative, the magnetic axis shifts outward along the major radius and the toroidal current becomes hollow at the midplane on the inner side of the toroidal plasma.14,15 This hollowness of the toroidal current profile becomes 
more pronounced as the pressure profile is made broader relative to the width of the toroidal magnetic well by increasing $M$ over $N$ in Eqs. (4) and (5). A small negative toroidal current forms near the inner wail when, for example, $C<-0.2$ for $N=1$ and $M=2$. Under these conditions, the negative diamagnetic term $I^{\prime}(\psi)$ balances much of the pressure term $R^{2} p^{\prime}(\psi)$ on the average, but because of the radial dependence of the pressure term, the diamagnetic term overcompensates at the inner edge of the toroid. A second magnetic axis appears from the inner wall if the depth of the magnetic well is increased further $(C<-0.55$ for $N=1$ and $M=2)$.

As the toroidal current profile becomes more peaked at the magnetic axis in equilibria with highly elongated walls, the flux surfaces near the magnetic axis become more circular.16-19 The elongation of the plasma is determined by image currents in the walls, and these image currents become less effective as the toroidal current becomes more concentrated. The elongation of the flux surfaces in the neighborhood of the magnetic axis relative to the elongation of the wall is represented here by (b/a) axis' (b/a) wall, where $b$ and $a$ are the half-height and half-width of a flux surface. The ratio $(b / a)_{a x i s} /(b / a)_{\text {wall }}$ decreases as the wall is elongated if $(b / a)$ axis $/(b / a)$ wall is less than unity, but the ratio increases as the wall is elongated if $(b / a)_{a x i s} /(b / a)$ wall is greater than unity. The relative shrinking of $(b / a)_{\text {axis }} /(b / a)$ wall with $(b / a)_{\text {wall }}$ becomes more pronounced with more peaked current profile, as illustrated in Fig. 2 . The properties of the particular equilibria chosen for this study of MHD instabilities are presented in Table 1 . Here, the poloidal beta, $B_{\text {pol' }}$, is defined by Eqs. A.19 to A.21 in the Appendix, and $\Delta / a$ represents the shift of the magnetic axis from the geometric center of the domain, 
relative to the minor radius of the wall. The pressure profiles for these four cases are almost the same, while the toroidal current and toroidal magnetic field profiles differ from each other significantly, as illustrated in Fig. 1. Note that both diamagnetism $\left(\beta_{\text {pol }}>1\right)$ and hroad current profile have the effect of elongating the inner flux surfaces. The combined effects of diamagnetism and broad current profile make the inner flux surfaces in case 4 more elongated than the outer flux surfaces and the wall.

The q-value and average beta of these equilibria are subsequently determined by scaling the equilibria as described in the Appendix. This scaling leaves the geometry of all the flux surfaces, their profile, and the poloidal beta completely unchanged. The q-value profile is changed only slightly.

\section{LTNEAR INSTABILITY}

Fixed-boundary MHD instabilities with one wavelength around the toroid $(n=1)$ are investigated here using the Oak Ridge linear initialvalue MHD instability code.12,20 The effects of clongation, breadth of the toruidal current profile, and diamagnetism are studied as they relate to the growth rate of the fastest growing $n=1$ MHD instability, the spatial structure of the eigenfunctiono, and the volume-averaged beta at marginal stability. Results are presented primarily for the four cases described in the last section. These results are found to be representative of a broad class of similar equilibria. 
A. Growth rates

Growth rate curves are presented in Figs. 3-6 for the four cases described in the last section, with three elongations of the wall, $\mathrm{b} / \mathrm{a}=$ 2, 3, 4, on each graph. For each curve, the growth rate is plotted as a function of $q_{c}$, the q-value at the magnetic axis, which is computed and then varied by scaling the equilibria as described in the Appendix. Under this scaling procedure, the geometric shape of the flux surfaces and the form of the profiles remain unchanged. The growth rate is normalized to the Alfven transit time across the minor radius of the plasma $\left(\gamma_{A} \equiv\right.$ $\left.\mathrm{B}_{\phi} / \sqrt{\mu_{0} \rho} \mathrm{a}\right)$. Only instabilities wi.th one wavelength around the toroid and, of those, only the instabilities with the lowest poloidal harmonic structure are considered here. It is possible, for example, that there are additional disconnected regions of instability at larger q-values which do not appear on these illustrations. However, the instabilities with a higher toroidal mode number and a higher q-value have a more complicated spatial structure which cannot be accurately computed with the grids and computer time available. This is also a problem for eigenvalue codes, such as $\mathrm{PESH}^{21}$ and ERATO, 22 as well as initial value codes such as our own. A sample of convergence studies with finer grids is discussed later in this paper (Fig. 7).

In every case, the growth rates of the instabilities studied increases monotonically with elongation. The increased growth rate is most pronounced in the cases with diamagnetism and broad current profile.

There is a dramatic change in the range of the instability as a function of q-value as the plasma is made diamagnetic with broad current 
profile. Compare, for example, Figs. 3 and 6 . Note that the ordinate in Fig. 6 spans a much wider range of q-values than the corresponding ordinates in Figs. 3-5. The marginal stability points are of order unity for all the elongations in the paramagnetic peaked-current profile case presented in Fig. 3. (If there is a tail on the b/a $=4$ case in Fig. 3, as there is in Figs. 4 and 5 , it is below the reliable resolution $\gamma=5 \times 10^{-3}$ of our computer code.) However, no marginal point at a11 could be found for the broad-current diamagnetic case of Fig. 6, and there are strong instabilities present at q-values well above unity. The cases studied in Figs. 4 and 5 show evidence of a transition as the toroidal current profile is made broad [by changing the powers $N$ and $M$ in the source functions of the Grad-Shafranov equation Eqs. (1)-(5)] or as the plasma 1s made diamagnetic [by changing coefficient C in Eq. (5)] with peaked-current profile, especially in the highly elongated case $\mathrm{b} / \mathrm{a}=4$. This transition emerges first as a tail on a growth rate curve that would otherwise intersect the ordinate at q-axie ncar unity, dul then as a shitt of the entire growth rate curve to higher q-values.

The range of unstable q-values and the magnitude of the growth rates depend more strongly on the elongation of the inner flux surfaces thall un the diamagnetism, for the cases we have studied. This sensitivity to elongation is illustrated in Fig. 8 where growth rate curves are presented for a variety of paramagnetic, diamagnetic, peaked current, and broad current cases, all with $(b / a)_{\text {wall }}=3$. Except for the two curves labeled $(\mathrm{b} / \mathrm{a})_{\mathrm{axis}} /(\mathrm{b} / \mathrm{a})_{\text {wall }}=0.80$ and 0.88 , the magnitudes of the growth rates and the range of the unstable q-values both increase monotonically with 
elongation of the inner flux surfaces. The one exception illustrates the milder effect of diamagnetism.

When a finer $35 \times 67$ grid is used for the computation of instabilities instead of the standard $35 \times 35$ grid, individual growth rates are decreased by as much as $10 \%$, but the form of the growth rate curves remain unchanged, as illustrated in Fig. 7. In particular, the tails on the growth rate curves in Figs. 4 and 5 remain, and there is the same general transition from kink to ballooning behavior.

\section{B. Eigenfunctions}

Velocity arrow plots and perturbed pressure contours are shown in Fig. 9 at cross sections of the plasma one quarter of a wavelength apart for cases 2,3 , and 4 with q-values close to the respective marginal points. The marginal eigenfunction for case 1 (not shown) is similar to that of case 2. This eigenfunction corresponds to an $m=1$ kink instability whose spatial extent shrinks down to the magnetic axis as the q-value is raised to the marginal point. In case 3, where the marginal q-value is just above unity, the marginal eigenfunction has the character of an $\mathrm{m}=2$ instability (with four vortex cells and two positive and two negative perturbed pressure peaks) which have a broader spatial extent. In the broad-current diamagnetic case 4, where the marginal q-value is well above unity, the instability has a strongly ballooning character $23-27$ with a high poloidal mode number concentrated away from the magnetic axis. The ballooning character of the instability is made evident from the fact that the perturbed pressure is strongest at the outer edge of the toroidal 
plasma (each case has an aspect ratio of four, with the center line to the left). It follows that the abrupt increase in the marginal q-value with broad-current diamagnetic equilibrium is accompanied by the onset of a strong ballooning character to the instability near the marginal point:

Elongation of the plasma cross section has the effect of greatly enhancing the ballooning character of the marginal instability, as shown in Fig. 10. Perturbed pressure contours are shown here at two cross sections of the plasma for a broad-current diamagnetic equilibrium, corresponding to case 4 , with $(b / a)_{\text {wall }}=1.5,2$, and 3 .

In summary, then, a strongly ballooning character to the marginal instability and a shift of the marginal q-value well above unity sets in for highly elongated equilibria $(b / a)_{\text {wall }}$ with a broad toroidal current profile as soon as the plasma becomes diamagnetic $\left(1<\beta_{\text {pol }}<2\right)$.

C. Maximum stable beta

It is clear that a compromise must be struck if the volume-averaged beta $\left(\langle\beta\rangle \equiv\langle p\rangle / \frac{1}{2 \mu_{0}} B_{\phi O}^{2}\right)$ is to be increased by elongating the plasma cross section, increasing diamagnetism, or making the toroidal current profile broader. For example, if the edge of a paramagnetic plasma with peaked profile is elongated, the volume-averaged beta will decrease because the central flux surfaces, where the pressure is the highest, will remain essentially circular while the additional space near the ends of the elongated wall will largely be filled with low-pressure plasma. If the diamagnetism is increased or the current profile made broader in a highly elongated plasma, the inner flux surfaces become more elongated and the 
volume of the high pressure region increases to make more efficient use of the available space, but the marginally stable q-value increases dramatically so that the toroidal current and, therefore, the volume-averaged beta must be decreased substantially in order to ensure stability. Hence, the optimum volume-averaged beta is achieved with a broad current profile equilibrium that is either diamagnetic with moderate elongation or paramagnetic with high elongation.

For the equilibria used in this paper, with an aspect ratio of four and variable elongation, the values of poloidal beta (defined in the Appendix), q-value at the magnetic axis, ratio of elongations (b/a) axis' (b/a) wall, and volume-averaged beta near the marginal point are presented in Table II. Since it is not always possible to make an accurate extrapolation of each growth rate curve to the marginal stability point, all the values listed in Table II refer to an extrapolation of the growth rate curves to the growth rate value $\gamma=0.007$. This is nominally the lower bound on the accuracy of our computer code with the grid size and time available. The grid size is relevant because the marginally stable eigenfunctions tend to have finer spatial structure than the eigenfunctions of more rapidly growing instabilities:

From Table II, it can be seen that the volume-averaged beta is only slightly increased by elongation in the peaked-current paramagnetic case. This is because the inner flux surfaces become only moderately elongated $\left[(b / a)_{a x i s}=2.08\right.$ when $\left.(b / a)_{\text {wall }}=4\right]$ at nearly fixed $q$-value on axis so that the pressure on axis rises while the fraction of space wasted on lowpressure plasma increases at nearly the same rate. Better results àre obtained in the broad-current paramagnetic example of case 2 . Here, the 
central flux surfaces elongate together with the elongation of the wall. However, the maximum volume-averaged beta (3.0\%) is achieved with $(\mathrm{b} / \mathrm{a})_{\text {wall }}=3$ because the marginal q-value at the magnetic axis rapidly increases with further elongation.

In the peaked-current diamagnetic example of case 3 , the rise in the elongation of the magnetic axis competes with the marginal q-value at the magnetic axis so that the volume-averaged beta remains essentially fixed up to elongations of $(b / a)_{\text {wall }}=3$. With further elongation of the wall, the central flux surfaces do not. elongate proportionally and the q-value on axis rises rapidly so that the volume-averaged beta decreases substantially.

The last entries in Table II pertain to an example of a broad-current diamagnetic plasma with less diamagnetism [smaller value of coefficient C in Eq. (5)] than in case 4 so that marginal stability points could be found for elongations of the wall up to three. In this case, the elonga-. tion of the central flux surfaces increases faster than the elnngatinn of the wall, so that very effective use is being made of the volume. However, the marginal q-value at the magnetic axis increases rapidly with elongation so that the volume-averaged beta decreases monotonically with elongatinn nf the wall.

From this study it follows that the maximum stable volume-averaged beta is achieved with highly elongated paramagnetic plasmas with broad toroidal current profile or with diamagnetic plasmas with moderate or little elongation. More detailed shaping and optimization of the profiles may further improve the volume-averaged beta but is not likely to reverse the trends indicated here. 
IV. INTERPRETATION AND COMPARISON WITH OTHER WORK

The research reported here is a computational study of fixed-boundary ideal MHD instabilities in a toroidal plasma with vertically elongated, perfectly-conducting rectangular walls. We are particularly interested in the transition between internal kink instabilities, which are generally localized to the region near the magnetic axis of the plasma, to ballooning modes, which are generally strongest around the outer edge of the toroidal plasma away from the magnetic axis. There are many other research papers reporting analytic, computational, or experimental studies of similar large-scale instabilities in elongated plasmas. In this section, we shall show how our results overlap with or can be interpreted in terms of a selection of these other papers. Only those references concerned with highly elongated cross sections ( $/ a>2)$, distributed current, and the ideal MHD model will be considered here.

We can estimate the conditions needed for the onset of pressuredriven instabilities near the outer edge of a highly elongated toroldal plasma from the following inequality

$$
\frac{2}{R}\left|\frac{d p}{d R}\right| \leqslant \operatorname{Max}\left[k_{\|}^{2} B^{2} ; \frac{1}{4} B_{p o 1}^{2}\left(\frac{1}{q_{L}} \frac{d_{L} L}{d R}\right)^{2}\right]
$$

where $R$ is the major radius, $k_{\|}$is the wave number parallel to the magnetic field, and

$$
q_{L} \equiv 2 b B_{\text {tor }} / 2 \pi R B_{\text {pol }}
$$


is a measure of the helical pitch (q-value) of the field lines along just the outer edge of the toroid. The expression on the left is the driving term due to a pressure gradient in the presence of toroidal curvature. The first expression on the right approximates the restoring force due to field line bending when the perturbation is a ballooning mode with onehalf wavelength equal to the length along the field lines around the outer edge of the toruid

$$
k_{\|} \simeq \frac{1}{\left(2 q_{L}^{R}\right)}
$$

The second term on the right is the restoring force due to shear stabilization of a perturbation localized near a mode rational surface, as in the derivation of the Suydam criterion. 27 A more exact combination of these stability criteria, involving both field line bending and shear stabilization, can be derived from the $n \rightarrow \infty$ limit of the energy principle, 28,29 as will be shown in a future publication. In Eqs. $(6)-(8)$, the elongated toroidal plasma is approximated by a cylindrical slab with the end affects neglected.

In the spirit of dimensional analysis, Eq. (6) yi.elds the approximate stability criterion

$$
\beta_{p \in 1}<\operatorname{Max}\left[\frac{1}{4} \frac{R}{a}\left(\frac{\pi}{b / a}\right)^{2} \frac{a_{p}}{a} ; \frac{1}{4} \frac{R}{a_{B}} \frac{{ }_{p}}{a_{B}}\right]
$$

where the scale lengths $a_{P}$ and $a_{B}$ are defined by

$$
a_{p} \equiv p_{\max } /\left|\frac{d p}{d R}\right|_{\max }
$$




$$
a_{B} \equiv \underset{\max }{B_{\text {max }}} /\left|\frac{d{ }_{p o 1}}{d R}\right|_{\max },
$$

and $\beta_{\text {pol max }}$ is

$$
\beta_{\text {po1 max }} \equiv P_{\max } / \frac{1}{2 \mu_{0}} B_{\max }^{2}
$$

When the gradient scale lengths are comparable to the minor radius of the plasma ( $a_{p} \sim a_{B} \sim a$ ), and the plasma is highly elongated, $b / a \geq 3$, it can be seen that the poloidal beta must be small, $\beta_{\text {pol } \max } \leqslant 1$, for stability of the $\mathrm{R} / \mathrm{a}=4$ plasma studied in this paper; the volume-averaged poloidal beta is even smaller. Elongation makes ballooning modes more unstabie because there is less restoring force due to field line bending as the connection length becomes longer. Under these conditions, there is little to be gained by further elongation. A similar conclusion was reached by Freidberg et al. using a surface current model of the plasma. Now let us consider other stability criteria that have appeared in the 1iterature. Lortz and $\ddot{N u h r e n b e r g}^{3}$ have evaluated a rigorous pair of necessary and sufficient stability criteria in the neighborhood of the magnetic axis for a toroidal plasma with any cross sectional shape and poloidal beta. They found favorable stability for highly elongated (at the magnetic axis) diamagnetic plasmas with $D$ shape (triangularity pointing away from the toroidal center line) for moderate q-values at the magnetic axis. We find that highly elongated, diamagnetic plasmas are violently unstable even at large q-values. The fact that the shape of our boundary conditions unavoidably produces flux surfaces with unfavoratle triangularity (with the flat eide pointing outward) probably 
contributes to this instability. However, a detailed study of the driving term for the most unstable mode has revealed that the region driving the instability shifts outward away from the magnetic axis, and the instability becomes predominantly pressure driven rather than parallel current driven as the plasma is made more elongated and diamagnetic. Therefore, as Lortz and Nuhrenberg pointed out, neither a necessary nor a sufficient stability criterion evaluated at the magnetic axis can be used to find instabilities such as ballooning modes, which are driven in regions away from the magnetic axis.

In our study of the instability driving terms, we evaluated the last two terms (separately) of Eq. (2) in Todd et al. 23 using the instability eigenfunctions determined by our initial value instability code. For the peaked profile paramagnetic equilibrium (case 1 ) with $b / a=3$, the kink term is much larger than the ballooning term and both are localized near the magnetic axis. The broad-current paramagnetic equilibrium (case 2) shows a mix of parallel current and pressure-driven terms. The currentdriven term is much larger than the pressure-driven term on the plane where the instability velocity field is mainly in the vertical direction $(\phi=0$ in Fig. 9). However, the current- and pressure-driven terms are more comparable a quarter of a wavelength around the toroid where the velocity field has a larger component along the major radius. In a toroidal environment, the mix of driving terms can vary around each flux surface.

The kink term dominates the ballooning term in the peaked-current diamagnetic equilibrium (case 3). Both kink and ballooning terms are 
localized near the magnetic axis, but the spatial distribution of the ballooning term is weighted more toward the outer edge of the toroid.

In the broad-current diamagnetic equilibrium (case 4), in which the instability had a strongly ballooning character, both the kink and the ballooning driving terms are distributed off the magnetic axis near the outer wall of the toroid. The pressure-driven term is several times stronger than the kink term. Again there is a strong dependence on the toroidal angle. From these observations, it can be seen how important the pressure-driven term can be in the region away from the magnetic axis for the stability of diamagnetic toroidal plasmas with broad current profiles and elongated cross sections.

The results of Laval, Pellat, and Soulélo and Grossmann, Tataronis, and Weitzner ${ }^{11}$ are primarily concerned with free-boundary instabilities, which are not considered in this paper. Assuming $\beta_{\text {pol }} \sim 0$ (1), large q-value at the edge of the plasma and large aspect ratio, Laval, Pellat, and Soule find that stability is improved with more peaked-current profile, in general agreement with our results. There is no evidence, however, that they were observing a transition between kink and pressure-driven ballooning behavior. In the work of Grossmann, Tataronis, and Weitzner, 11 the assumption that the elongation $\mathrm{b} / \mathrm{a}$ scales like $\varepsilon^{-1 / 2}$, where $\varepsilon \equiv \mathrm{a} / \mathrm{R}$ is the reciprocal of the aspect ratio, restricts their attention to relatively broad-current profiles where the inner flux surfaces are highly elongated. Under these conditions, there is general agreement that the equilibria are unstable.

There has been a series of experiments ${ }^{29-34}$ in which highly elongated diamagnetic toroidal plasmas have been formed by shock heating and maintained for many Alfvén transit times. In the Belt Pinch experiments, 30-35 
large-scale stability is observed even in highly elongated discharges $\left[(b / a)_{\text {wall }} \sim 5\right.$ to 10$\left.)\right]$, when the q-value at the wall is greater than three. An $m=1$ instability is observed at lower q-values, although this instability is observed to saturate and then go away when the q-value decreases below and then increases above the instability limit during the discharge. In high density stable discharges, filamentary structures are observed around the outer side of the plasma coincident with the helical pitch of the magnetic field lines. 30 The filaments decay as the plasma elongation contracts and as the temperature and diamagnetic currents decay. These observations are consistent with the picture that ballooning modes begin to grow and cause a filamentation of the outer side of the plasma while the plasma is diamagnetic and elongated with a broad toroidal current profile; but these ballooning modes saturate and decay as the plasma pressure and elongation decay. This interpretation is certainly reasonable in Belt Pinch I investigated by Krause, in which the plasma temperature decayed to $<5 \mathrm{eV}$ within $10 \mathrm{sec}$. In the larger and more optimized device, Belt Pinch IIa, the filamentary structure is observed only in the high compression case. ${ }^{32}$ The filaments are not observed optically in the low compression discharges in Belt Pinch IIa, but the experimenta1 observations do not rule out the possibility of ballooning modes. ${ }^{32}$ In particular, fluctuating magnetic fields and enhanced energy losses are observed under these conditions.

\section{CONCLUSION}

Under the influence of internal $\mathrm{n}=1 \mathrm{kink}$ and ballooning modes in elongated toroidal equilibria, the maximum stable volume-averaged beta 
is achieved with a broad-current profile and either a paramagnetic plasma $\left(\beta_{\text {pol }}<1\right)$ with a highly elongated cross section $(b / a>2)$ or a diamagnetic plasma $\left(\beta_{\mathrm{pol}}>1\right)$ with only a mildly elongated cross $\operatorname{section}(\mathrm{b} / \mathrm{a}<2)$. These observations are summarized in Table II. Internal ballooning modes are most easily observed in highly elongated diamagnetic plasmas with q-axis $>1$ and a broad-current profile. While parallel current has some destabilizing effects on these modes, the dominant driving mechanism is the pressure gradient in the toroidal curvature of the magnetic field lines.

Theoretical evidence suggests that these pressure-driven instabilities near the edge of the plasma can be very sensitive to the boundary conditions. If they are present under fixed-boundary conditions, they will certainly be present and will probably be much stronger under freeboundary conditions. Also, it is likely that a resistive instability theory will indicate the onset of resistive ballooning instabilities resulting in a deterioration of plasma confinement at lower pressures than the onset of the ideal instability. Therefore, the fixed-boundary ideal MHD theory presented here probably predicts an upper bound on the pressure we can expect to confine in elongated toroidal plasmas.

\section{ACKNOWLEDGMENTS}

This research was sponsored jointly by the University of Tennessee under contract DOE EY-76-S-052598 and the Office of Fiston Eutery (ETM), U.S. Department of Energy under contract W-7405-eng-26 with the Union Carbide Corporation. 
APPENDIX

Several calculations concerning the equilibrium will be considered in this Appendix. In particular, the method for computing the q-value at the magnetic axis given data on a discrete grid will be derived, and the scaling technique used to obtain the growth rate curves in this paper will be presented. Finally, a collection of definitions of beta, poloidal beta, and other equilibrium parameters nsed in this paper will be 1isted for refélente.

In the course of doing computational work on MHD instabilities, it has been found useful to have an expression for the q-value at the magnetic axis for any axisymmetric toroidal equilibrium given the flux or magnetic field at only a discrete set of grid points. The usual methods of computing the q-value, involving line integrals or area integrals within flux surfaces, break down near the magnetic axis.

The derivation proceeds by fitting a polynomial approximation (i.e., a truncated laylor series) to the poloidal flux array at the grid points in the neighborhood of the magnetic axis. The q-value is then determined analytically from the expression

$$
q(\psi)=d \psi_{\text {tor }} / d \psi_{\text {pol }}
$$

where $\psi_{\text {tor }}$ is the tornidal flux given by

$$
\psi_{\text {tor }}(\psi)=\int_{\psi^{\prime}>\psi_{\text {pol }}} \mathrm{dRdy} \mathrm{B}_{\phi},
$$


and $\psi_{\text {pol }}$ is the poloidal flux which is proportional to the stream function $\psi=\psi_{\text {pol }} / 2 \pi$ used in the Grad-Shafranov equation.

Since a Cartesian grid is used in both the equilibrium and instability codes, we make a series expansion the stream function $\psi$ near the magnetic axis using a local Cartesian coordinate system $(x, y)$ centered at the magnetic axis $\left(x_{a}, y_{b}\right)$

$$
\psi=\psi_{a}+\frac{1}{2} \psi_{a 20}\left(x-x_{a}\right)^{2}+\frac{1}{2} \psi_{a 02}\left(y-y_{a}\right)^{2}+\ldots .
$$

Linear terms are absent because $\psi$ assumes its maximum value at the magnetic axis. Transforming to a local polar coordinate system around the magnetic axis $(r, \theta)$ and then to flux-angle coordinates $(\psi, \theta)$, the expression for the toroidal flux takes the form

$$
\psi_{\text {tor }}(\psi)=\int_{\psi}^{\psi} a d \psi^{\prime} \int_{0}^{2 \pi} d \theta^{\prime} \frac{I\left(\psi^{\prime}\right) r \partial r / \partial \psi^{\prime}}{1+\alpha\left(x_{a}+r \cos \theta^{\prime}\right)}
$$

where $R=R_{0}(1+\alpha x)$ and $R_{0}$ is the major radius to the geometric center of the domain. The q-value per unit wavelength around the toroid, nq, then takes the form

$$
\mathrm{nq}=\frac{\mathrm{I}(\psi)}{\lambda} \int_{0}^{2 \pi} d \theta \frac{\mathrm{r} \partial \mathrm{r} / \partial \psi}{1+\alpha\left(\mathrm{x}_{\mathrm{a}}+\mathrm{r} \cos \theta\right)}
$$

where

$$
\lambda=2 \pi \mathrm{R}_{0} / \mathrm{rl}
$$


Using $\mathrm{x}-\mathrm{x}_{\mathrm{a}}=\mathrm{r} \cos \theta$ and $\mathrm{y}-\mathrm{y}_{\mathrm{a}}=\mathrm{r} \sin \theta$, we can invert Eq., (A.3) to determine $r=r(\psi, \theta)$ up to second order in the expansion. Then, to find the q-value at the magnetic axis, take the limit $\psi \rightarrow \psi_{a}$ and $r(\psi, \theta) \rightarrow$ 0 , and Eq. (A.5) reduces to

$$
\mathrm{nq}_{\mathrm{axis}}=\frac{I\left(\psi_{\mathrm{a}}\right)}{\left(1+\alpha \mathrm{x}_{\mathrm{Q}}\right) \lambda} \int_{0}^{2 \pi} \frac{\mathrm{d} \theta}{\psi_{\underline{Q} 02} \cos ^{2} \theta+\psi_{\mathrm{g} 02} \sin ^{2} \theta}
$$

The result of this standard integral is

$$
\mathrm{nq}_{\mathrm{axis}}=\frac{2 \pi \mathrm{B}_{\phi \text { axis }}}{\lambda\left(\psi_{\left.\mathrm{a} 20 \psi_{\mathrm{a} 02}\right)^{1 / 2}}\right.}
$$

In terms of the magnetic fields, the q-value per unit wavelength is

$$
\mathrm{nq}_{\mathrm{axis}}=\frac{2 \pi \mathrm{B}_{\phi} \text { axis }}{\lambda\left[\left(-\frac{\partial}{\partial \mathrm{R}} \frac{\mathrm{RB}_{\mathrm{y}}}{\mathrm{R}_{\mathrm{o}}}\right)\left(\frac{\partial}{\partial \mathrm{y}} \frac{\mathrm{RB}}{\mathrm{R}} \mathrm{R}_{\mathrm{o}}\right)\right]^{1 / 2}} .
$$

These expressions for the q-value depend upon only the elliptical distortion of the flux surfaces in the immediate neighborhood of the magnetic axis, independent of their triangularity or relative shift. If there were a cross product term $\psi_{11} x y$ in the series expansion (A.3) for the poloidal flux, then the local coordinate system could be rotated to eliminate this term, altering the coefficients $\psi_{\mathrm{a} 20}$ and $\psi_{\mathrm{a} 02}$ but preserving Eq. (A.6). For the specific example of a straight cylinder, uniform current equilibrium with elliptical cross section, 
$\psi=\psi_{a}+\frac{1}{2} \frac{J_{z_{0}}}{\mu_{0}} \frac{a^{2} b^{2}}{b^{2}+a^{2}}\left(\frac{x^{2}}{a^{2}}+\frac{y^{2}}{b^{2}}\right)$.

Eq. (A.6) yields the correct q-value

$$
\mathrm{nq}_{\text {axis }}=\frac{2 \pi}{\lambda} \frac{\mathrm{B}_{\mathrm{za}}}{\mu_{\mathrm{o} z a} \mathrm{a}_{\mathrm{z}}} \frac{\mathrm{a}^{2}}{\mathrm{ab}} \text {. }
$$

Now suppose the values of flux or magnetic field are given at only a discrete set of grid points and the magnetic axis does not necessarily fall on any of these points. The problem is to find the coordinates of the magnetic axis $\left(x_{a}, y_{a}\right)$ and the second order coefficients $\left(\psi_{a 20}, \psi_{a 02}\right)$ in the series expansion (A.3). One approach is to evaluate Eq. (A.3) at a number of grid points and then invert the resulting series of equations to find the unknowns. Another method is to make a separate series expansion around the grid point $\left(\mathrm{x}_{\mathrm{o}}, \mathrm{y}_{\mathrm{o}}\right)$ nearest the magnetic axis (the grid point where $\psi_{i j}$ assumes its maximum value) and then find the relation between the coefficients of the two series expansions. The coefficients of the second series expansion $\psi_{i j}$ are just the standard finite difference approximations to the spatial derivatives $\left(\partial / \partial_{x}\right)^{i}\left(\partial / \partial_{y}\right)^{j} \psi$, for example,

$$
\begin{aligned}
& \psi_{10}=[\psi(1,0)-\psi(-1,0)] / 2 \mathrm{dx} \\
& \psi_{20}=[\psi(1,0)-2 \psi(0,0)+\psi(-1,0)] /(\mathrm{dx})^{2} .
\end{aligned}
$$


The relations between the two sets of coefficients, up to second order, are

$$
\begin{aligned}
\psi_{a 20} & =\psi_{20} \\
\psi_{a 0 z} & =\psi_{02} \\
x_{a} & =x_{0}-\psi_{10} / \psi_{20} \\
y_{a} & =y_{0}-\psi_{01} / \psi_{02} .
\end{aligned}
$$

For an estimate of the error, use

$$
\begin{aligned}
\psi_{a 20} & =\psi_{20}-\psi_{30} \delta_{\mathrm{x}}-\psi_{21} \delta_{\mathrm{y}} \\
& -\psi_{40} \delta_{\mathrm{x}}^{2}-\psi_{31} \delta_{\mathrm{x}} \delta_{\mathrm{y}}-\psi_{22} \delta_{\mathrm{y}}^{2}+0\left(\delta^{3}\right)
\end{aligned}
$$

where

$$
\begin{aligned}
\delta_{\mathrm{x}} \equiv \mathrm{x}_{\mathrm{a}}-\mathrm{x}_{\mathrm{o}}=-\frac{1}{\psi_{20}}\left[\psi_{10}\right. & +\frac{1}{2} \psi_{30} \delta_{\mathrm{x}}^{2}+\psi_{21} \delta \delta^{\delta} \mathrm{y} \\
& \left.+\frac{1}{2} \psi_{12} \delta_{\mathrm{y}}^{2}+O\left(\delta^{3}\right)\right]
\end{aligned}
$$

with corresponding symmetric relations for $\psi_{a \cup z}$ and $\delta_{y}$.

Now consider the equilibrium scaling relation used to obtain the growth rale curves in this paper and in many other computational studies of MHD instabilities. It follows from the Grad-Shafranov equation (1) that the stream function $\psi$ can be scaled by a numerical factor $S$ 


$$
\psi_{\text {new }}=S \psi_{\text {old }}
$$

provided the pressure and diamagnetism are scaled by $\mathrm{s}^{2}$

$$
\mathrm{P}_{\text {new }}=\mathrm{S}^{2} \mathrm{P}_{\mathrm{old}}
$$

regardless of the functional form of $p(\psi)$ and $I(\psi)$. The geometry, the shape of the profiles, and the poloidal beta (as defined later in this section) remain unchanged by this scaling. The poloidal magnetic field, toroidal current, and toroidal current density all scale like $S$; the value of beta scales like $\mathrm{s}^{2}$. This scaling technique is applicable to any MHD equilibrium with continuous symmetry (straight cylinder, axisymetric toroid, or equilibrium with helical symmetry).

Since the shape of the flux surfaces remains unchanged by this scaling, it follows that the q-value at any point in space (on any flux surface) scales like

$$
q_{\text {new }}=q \sqrt{\left(1-S^{2}\right)\left(R B_{\phi}\right)_{\text {edge }}^{2}+s^{2}\left(R B_{\phi}\right)^{2}} /\left.S R B_{\phi}\right|_{\text {old }}
$$

Given any equilibrium with $\mathrm{q}_{\mathrm{old}}$ determined at any reference point (the q-value at the magnetic axis has been used throughout this paper) the scale factor needed to obtain a new equilibrium with $q_{\text {new }}$ is

$$
S^{2}=I_{\substack{\text { edge } \\
\text { new }}}^{2} /\left[\begin{array}{l}
I_{\text {elge }}^{2} \\
\text { old }
\end{array}\right.
$$

Finally, we present some of the definitions used throughout this paper. The volume-averaged beta i.s given by the volume-averaged pressure 
divided by the magnetic pressure associated with the vacuum toroidal magnetic field above the geometric center of the domain. The poloidal beta is defined in terms of the diamagnetism of the plasma

$$
\beta_{p \cap 1 .} \equiv 1+\mu_{p}
$$

where

$$
\mu_{p}=\left\langle\left(\frac{R B_{\phi}}{R_{0}}\right)_{\text {edge }}^{2}-\left(\frac{R B_{\phi}}{R_{0}}\right)^{2}\right\rangle / \frac{B_{\text {pol. }}^{2}}{-}
$$

and

$$
\overline{\beta_{p o 1}^{2}}=\oint: d \ell B_{p o 1}^{2} / \oint d \ell
$$

Using this definition, the poloidal beta is identically equal to unity when there are no poloidal currents. 


\section{REFERENCES}

${ }^{1}$ T. Ohkawa and H. G. Voorhies, Phys. Rev. Lett. 22, 1275-1277 (1969).

${ }^{2}$ L. A. Artsimovich and V. D. Shafranov, JETP Lett. 15, 51-54 (1972).

${ }^{3}$ D. Lortz and J. Nïhrenberg, Nucl. Fusion 13, 821-827 (1973).

${ }^{4}$ J. M. Greene and J. L. Johnson, Phys. Fluids 5, 510-517 (1962).

${ }^{5}$ D. Lortz, Nucl. Fusion 13, 817-819 (1973).

${ }^{6} \mathrm{~J}$. P. Freidberg and F. A. Haas, Phys. Fluids 17, 440-446 (1974).

7B. M. Marder, Phys. Fluids 17, 447-451, 634-639 (1974).

${ }^{8}$ J. P. Freidberg and W. Grossmann, Phys. Fluids 18, 1494-1506 (1975).

${ }^{9}$ J. P. Freidberg, J. P. Goedbloed, W. Grossmann, F. A. Haas, PZasma

Physics and Controlzed Nuclear Fusion Research 1974 (IAEA, Vienna, 1975), Vo1. I, pp. 505-514.

${ }^{10}$ G. Laval, R. Pellat, and J. S. Soulé, Phys. Fluids 17, 835-845 (1974).

${ }^{11}$ W. Grossmann, J. A. Tataronis, and H. Weitzner, Phys. Fluids 20, 239-251 (1977).

${ }^{12}$ G. Bateman, W. Schneider, and W. Grossmann, Nucl. Fusion 14; 669-683 (1974).

130. Buneman, Stanford University Report SUIPR 294 (1969).

${ }^{14}$ V. S. Mukhovatov and V. D. Shafranov, Nucl. Fusion 11, 605-633 (1971).

$15 \mathrm{~J}$. D. Callen and R. A. Dory, Phys. Fluids 15, 1523-1528 (1972).

${ }^{16} \mathrm{~K}$. V. Hagenow and K. Lackner, Proc. 3rd Int. Symp. on Toroidal Confinement, Garching, 1973, F-7.

17 W. Feneberg and K. Lackrier, Nuc1. Fusion 13, 549 (1973).

${ }^{18}$ A. Kadish, Nucl. Fusion 13,756-757 (1973); A. Kadish and D. C. Stevens, Nuc1. Fusion 1.4, 821-829 (1974). 
${ }^{19} \mathrm{G}$. Becker, Nuc1. Fusion 14, 319-321 (1974).

$20 \mathrm{H}$. R. Hicks and J.W. Wooten, Computer Physics Communications 13 , 117-135 (1977).

${ }^{2 I}$ R. C. Grimm, J. M. Greene, and J. L. Johnson, in Methods in Computational Physics, edited by J. Killeen (Academic Press, N.Y., 1976), Vol. 16, pp. 253-281.

${ }^{22}$ D. Berger, L. C. Bernard, R. Gruber, and F. Troyon, Plasma Physics and Controlzed Nuclear Fusion Research 1976 (IAEA, Vienna, 1977), Vol. II, pp. $411-42 i$.

${ }^{23}$ A. M. M. Todd, M. S. Chance, J. M. Greene, R. C. Grimm; J. L. Johnson, and J. Manickam, Phys. Rev. Lett. $\underline{38}$, 826-829 (1977).

${ }^{24}$ G. Bateman and Y-K. M. Peng, Phys. Rev. Lett. 38, 829-832 (1977).

${ }^{25}$ A. Sykes, J. A. Wesson, and S. J. Cox, Phys. Rev. Lett. 39, 757-760 (1977).

${ }^{26}$ B. Coppi, Phys. Rev. Lett. 39, 939-942 (1977).

27 B. R. Suydam, Geneva Conference, Vol. 31, 157-159 (1958).

${ }^{28}$ D. Dobrott, D. B. Nelson, J. M. Greene, A. H. Glasser, M. S. Chance, and E. A. Frieman, Phys. Rev. Lett. 39, 943-946 (1977).

$29 \mathrm{~J}$. W. Connor, R. J. Hastie, and J. B. Taylor, Phys. Rev. Lett. 40, 396-399 (1978).

${ }^{30} \mathrm{H}$. Krause, Nucl. Fusion 15, 855-863 (1975).

$31_{0}$. Gruber and R. Wilhelm, Nucl. Fusion 16, 243-251 (1976).

${ }^{32}$ G. Becker, O. Gruber, H. Krause, F. Mast, R. Wilhelm, Proc. 8th European Conference on Controlled Fusion and Plasma Physics (Prague, 1977), Vol. I, p. 76 . 
${ }^{3}{ }^{3}$ K. H. Dippel, E. Graffman, F. Hoenen, L. Janicke, A. Kaleck, H. Kever, L. Koenen, A. Nicolai, U. Plantikow, J. Schlüter, PZasma Physics and Controlzed Nuclear Fusion Research 1976 (IAEA, Vienna, 1977), Vol. II, pp. $411-421$.

${ }^{34}$ D. Düchs, R. H. Dixon, and R. C. Elton, Proc. 2nd Topical Conference on Pulsed High-Beta Plasmas, W. Lotz, ed., Max-Planck IPP Report 1/127 (July, 1972) 111-114.

${ }^{35}$ A. W. Allen, F. L. Cochran, G. C. Goldenbaum, and P. C. Liewer, Phys. Rev. Lett. 39, 404-407 (1977). 
FIGURE CAPTIONS

Fig. 1. Equilibrium profiles for the four cases studied in this paper, with elongation $(b / a)_{\text {wall }}=3$. Flux contours over a cross section of the plasma are shown on the left. The toroidal current density and magnetic field at the midplane are shown on the right.

Fig. 2. Variation of the relative elongation at the magnetis axis as a function of the elongation at the wall for the four cases studied in this paper.

Fig. 3. Growth rate as a function of q-value at the magnetic axis for the peaked-current paramagnetic equilibrium of case 1 .

Fig. 4. Growth rate as a function of q-value at the magnetic axis tor the broad-current paramagnetic equilibrium of case 2 .

Fig. 5. Growth rate as a function of q-value at the magnetic axis for the peaked-current diamagnetic equilibrium of case 3 .

Fig. 6. Growth rate as a function of q-value at the magnetic axis for the broad-current diamagnetic equilibrium of case 4 . Note that the range of q-values extends much higher here than in Figs. 3-5.

Fig. 7. Growth rate curves as a function of q-value at the magnetic axis for different grid resolutions. The $35 \times 35$ is the standard used in the rest of the paper.

Fig. 8. Growth rate curves as a function of q-value at the magnetic axis for a variety of equilibria with different relative elongations of the flux surfaces near the magnetic axis.

Fig. 9. Spatial structure of the velocity fleld and the perturbed pressure, each shown at two cross sections a quarter of a wavelength apart, for instabilities near the marginal points of three cases studied in this paper. Contours for positive (solid lines) and negative (dntted lines) perturbed pressures are shown. From top to bottom, the instabilities exhibit an increasingly ballooning character.

Fig. 10. Perturbed pressure contours at cross sections a quarter of a wavelength apart for instabilities near the marginal point for the broad-current diamagnetic equilibrium corresponding to case 5 . From left to right, the elongation is increased while the aspect ratio is held fixed at $\mathrm{R} / \mathrm{a}=4$ (the center line is to the left). 


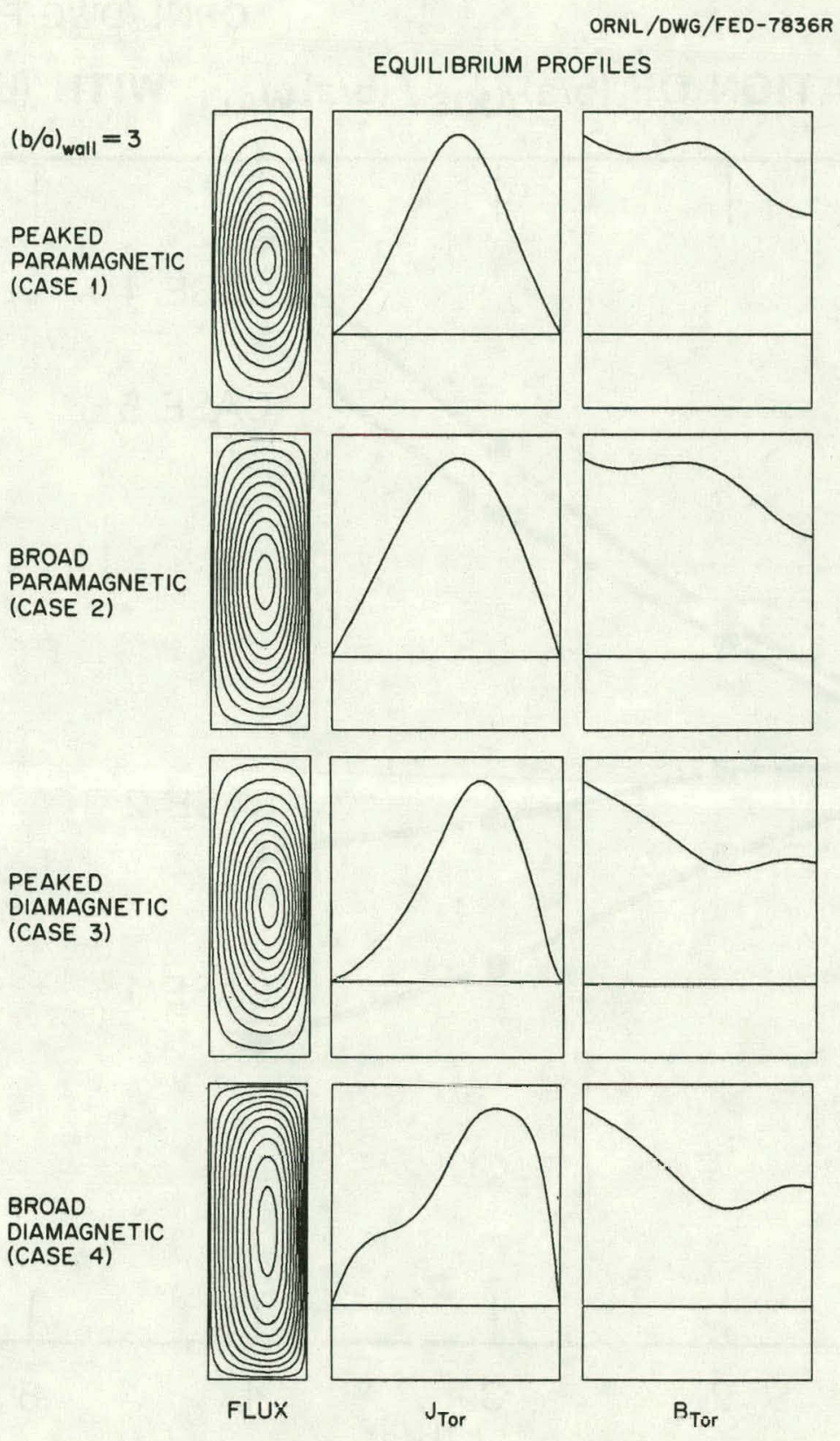

Fig. 1 . 
ORNL/DWG/FED - 78 - 431

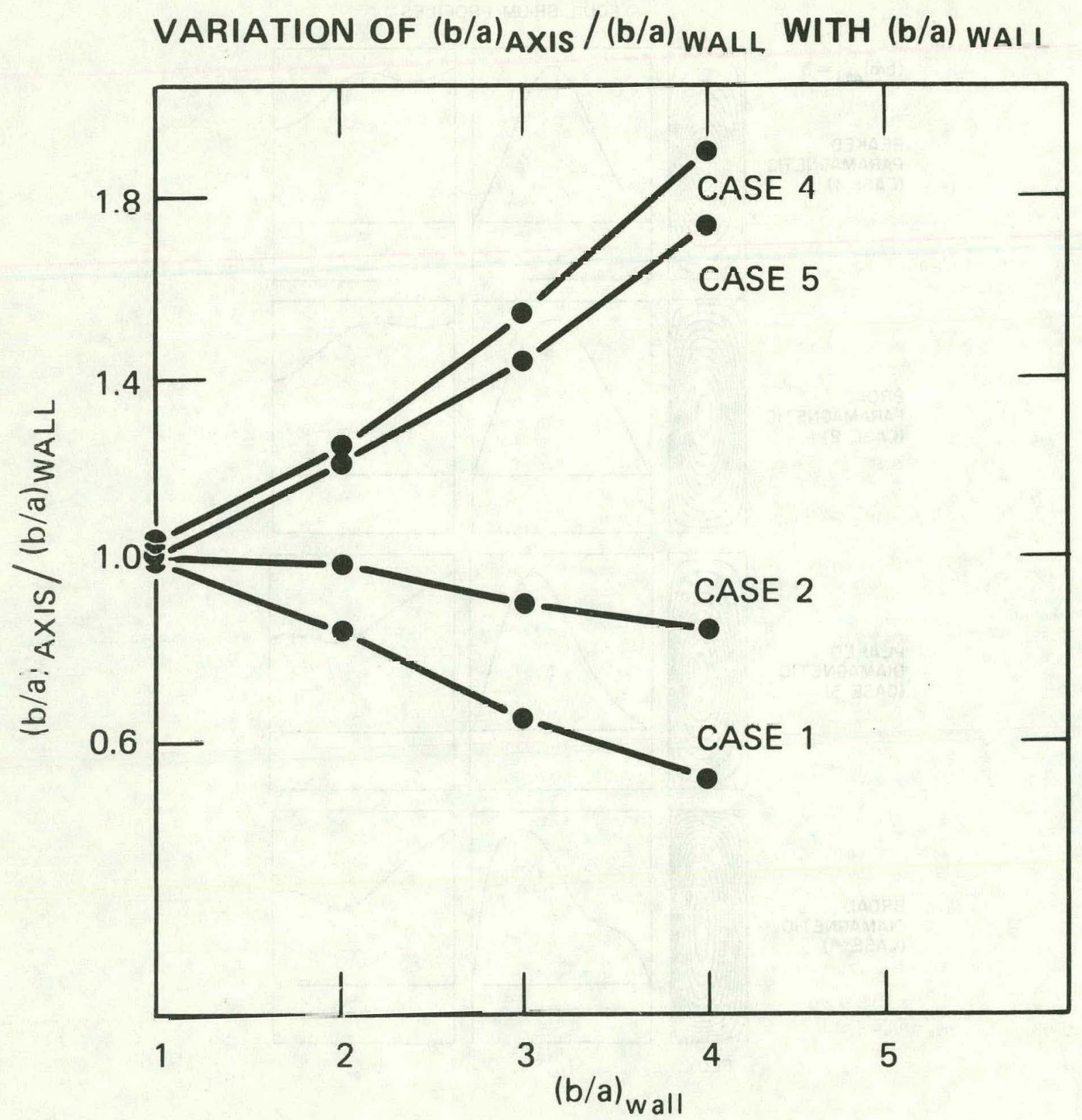

Fig. 2. 


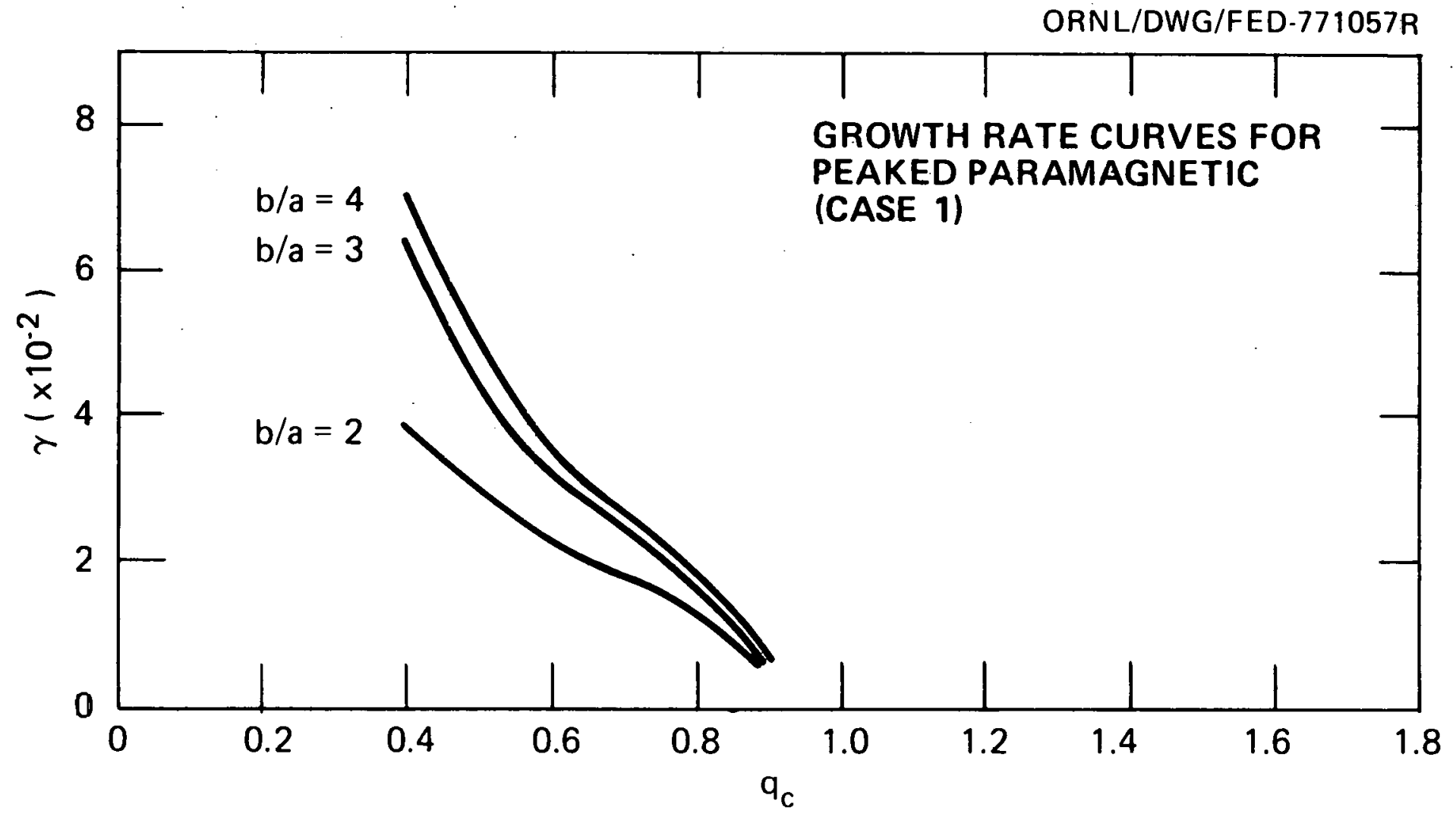

Fig. 3. 


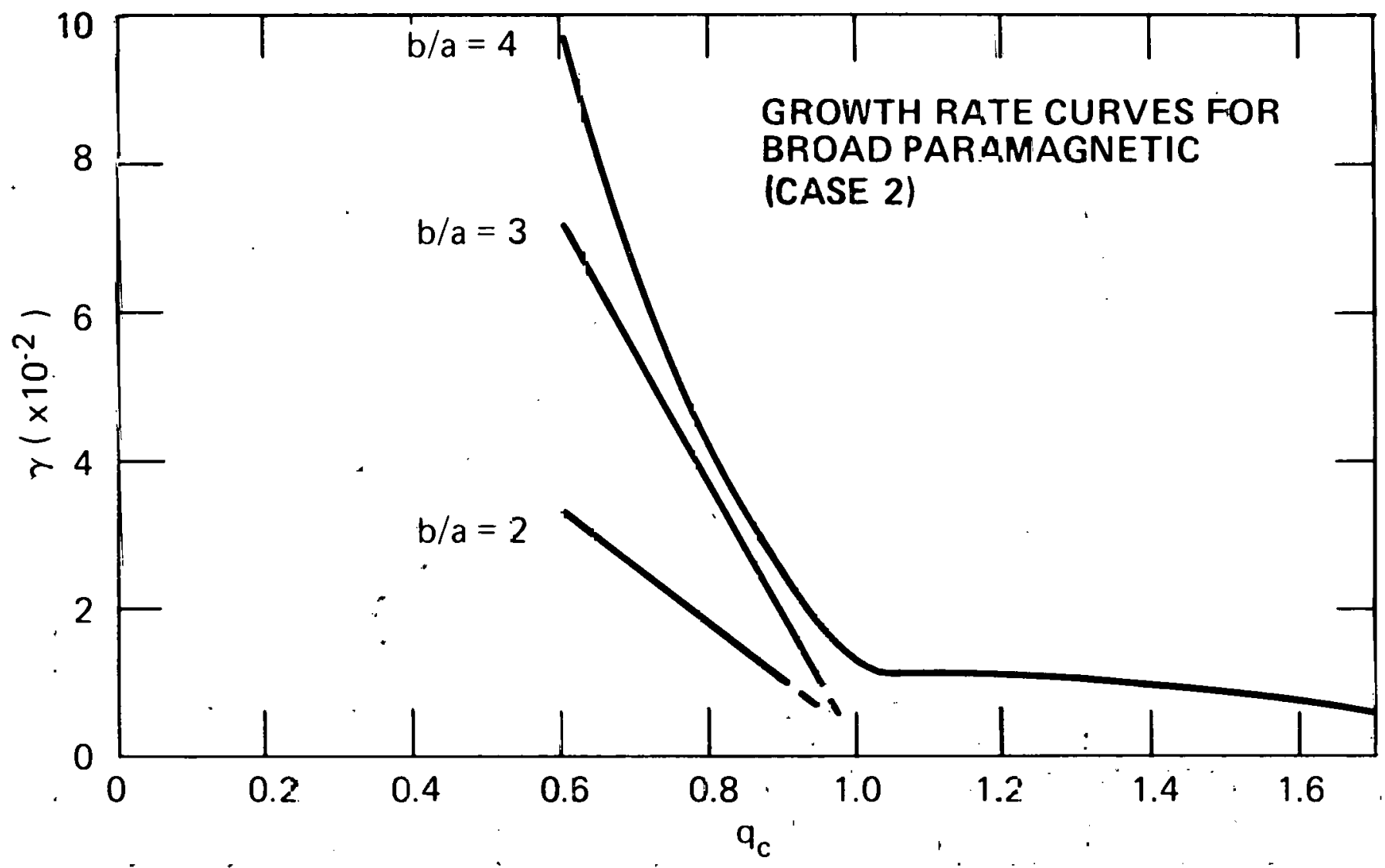

Fig. 4. 


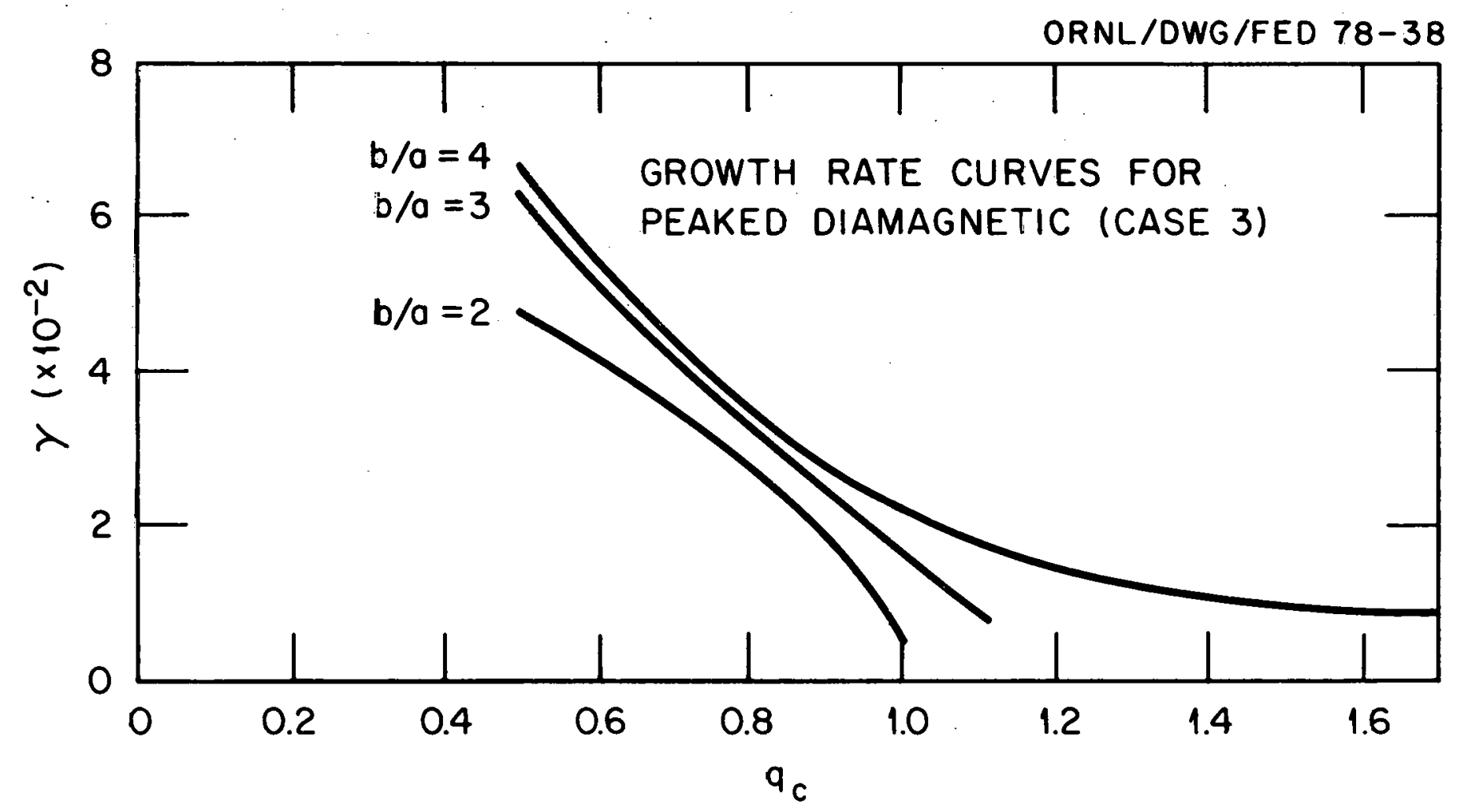

Fig. 5. 
JRNL/DWG/FED $78 \cdot 275$

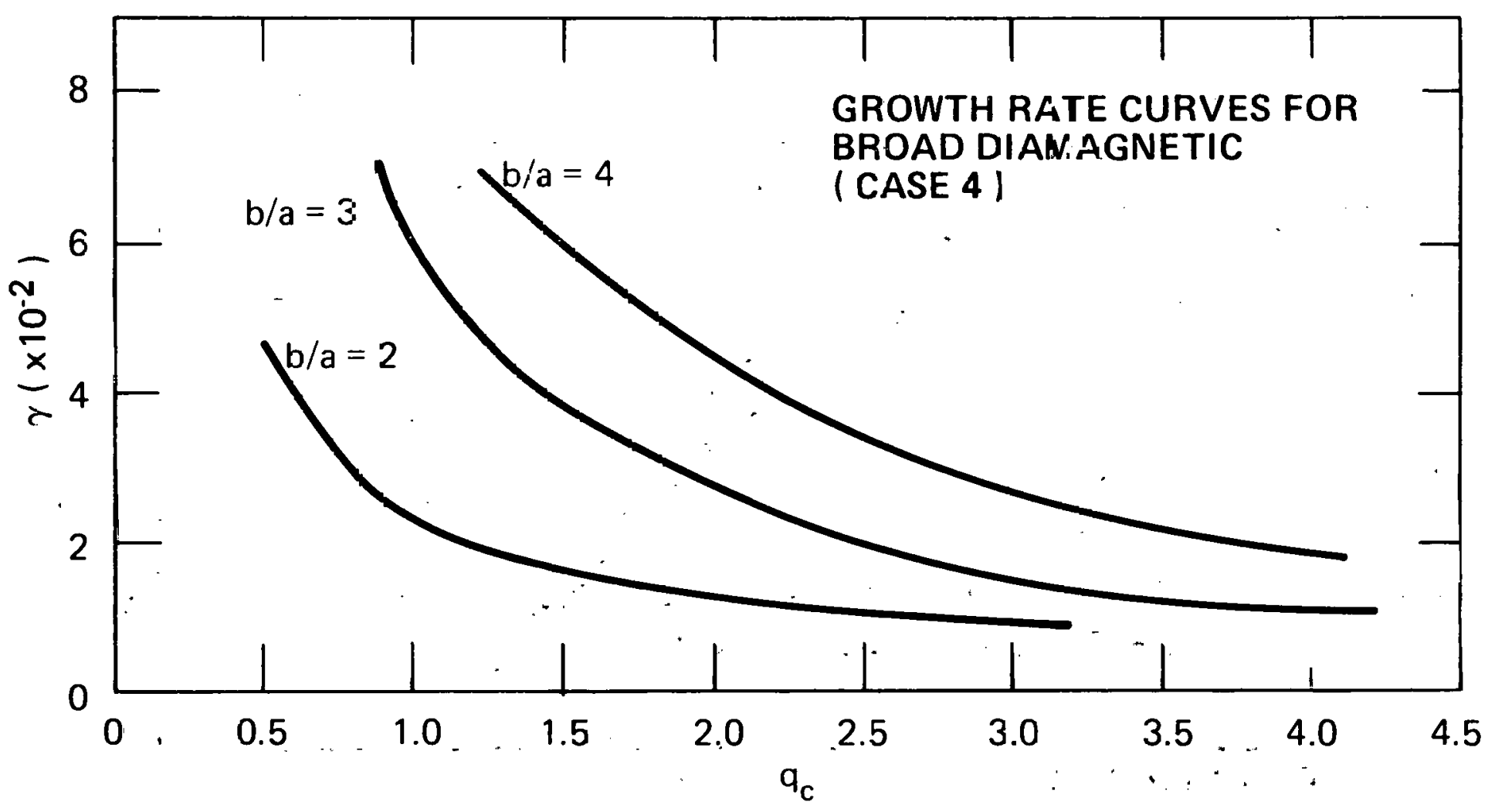

Fig. 6. 
ORNL/DWG/FED - 78 - 432

COMPARISON OF GROWTH RATES

WITH DIFFERENT GRID SIZE

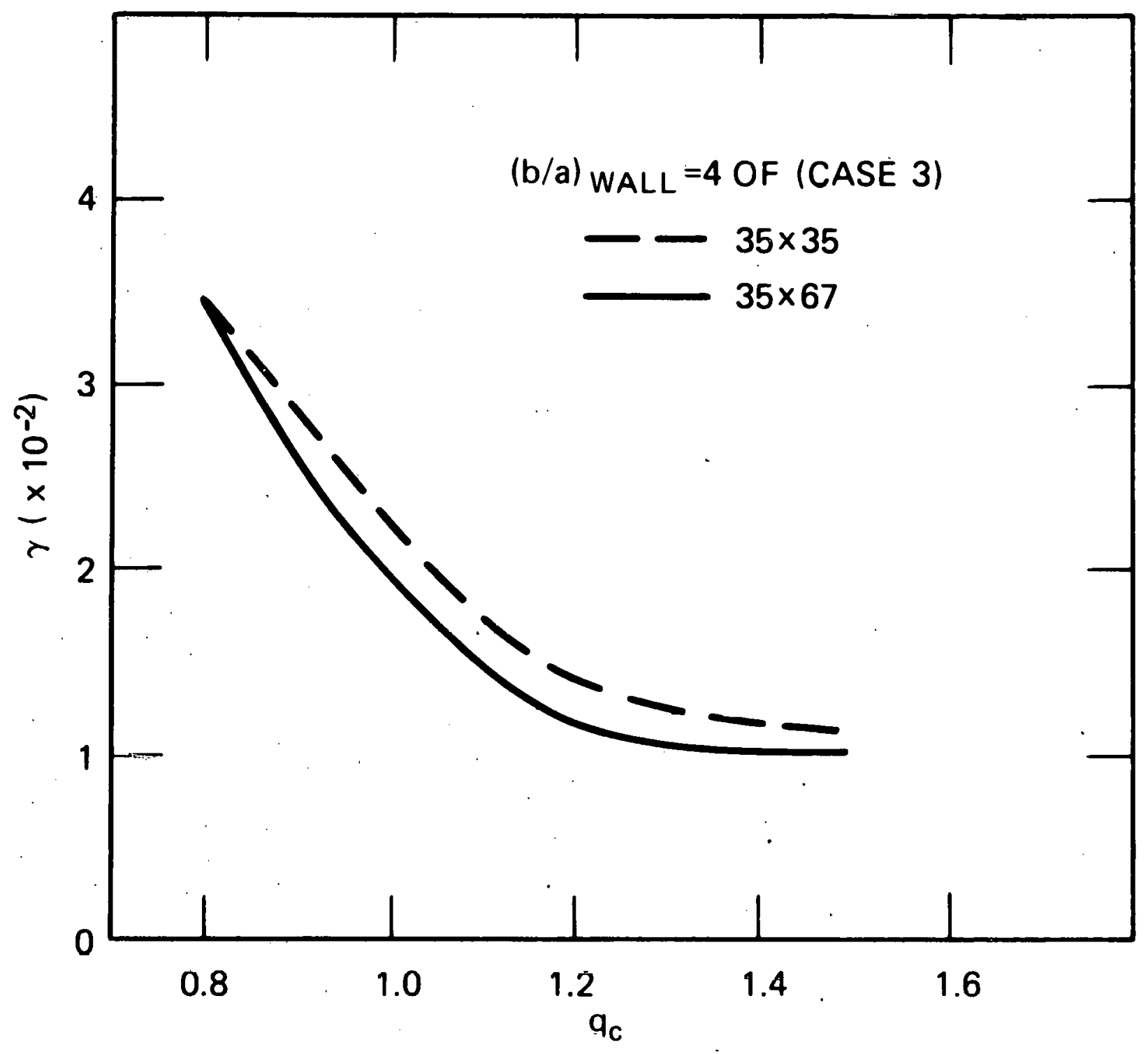

Fig. 7 . 


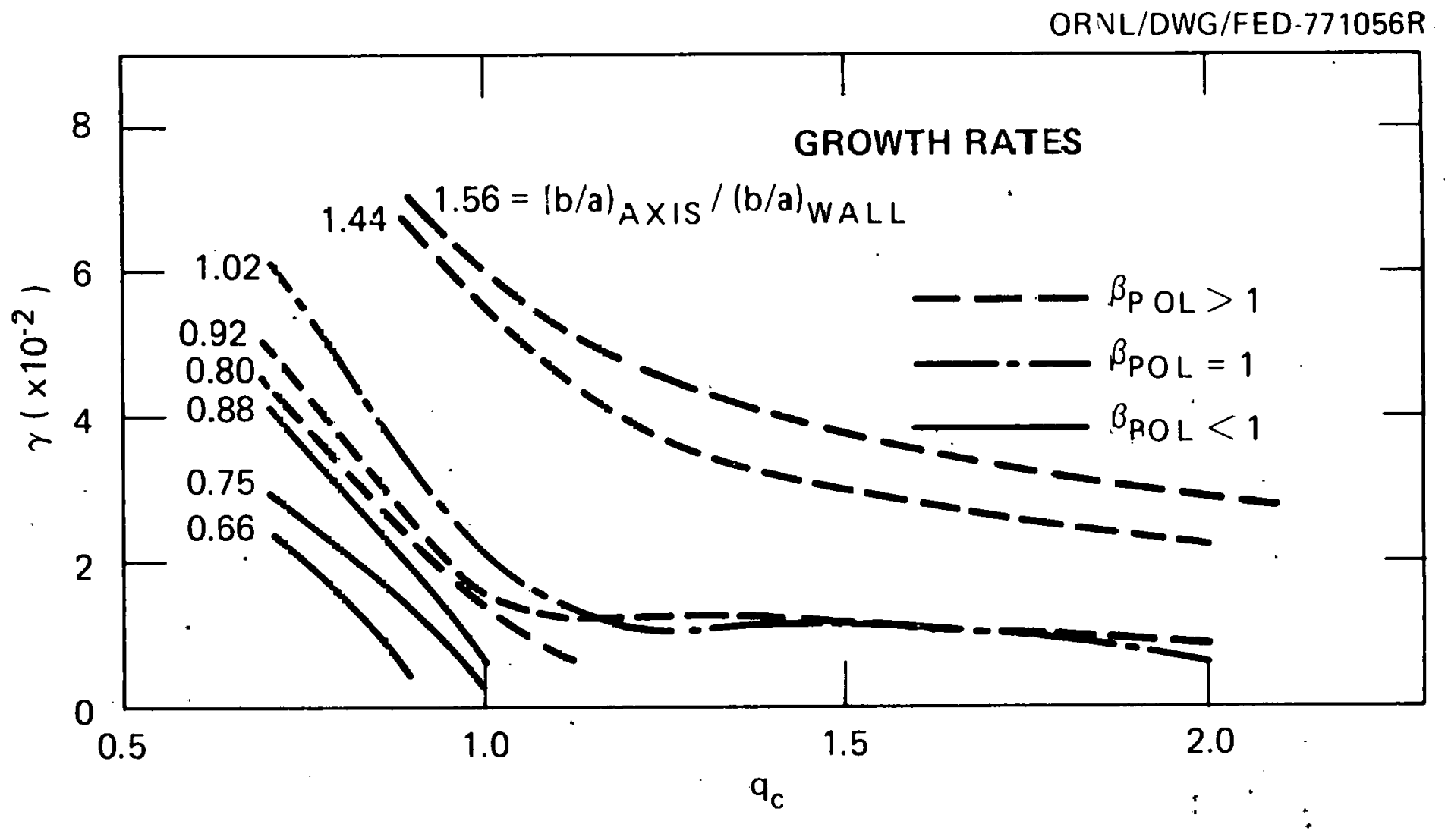

Fig. 8: 


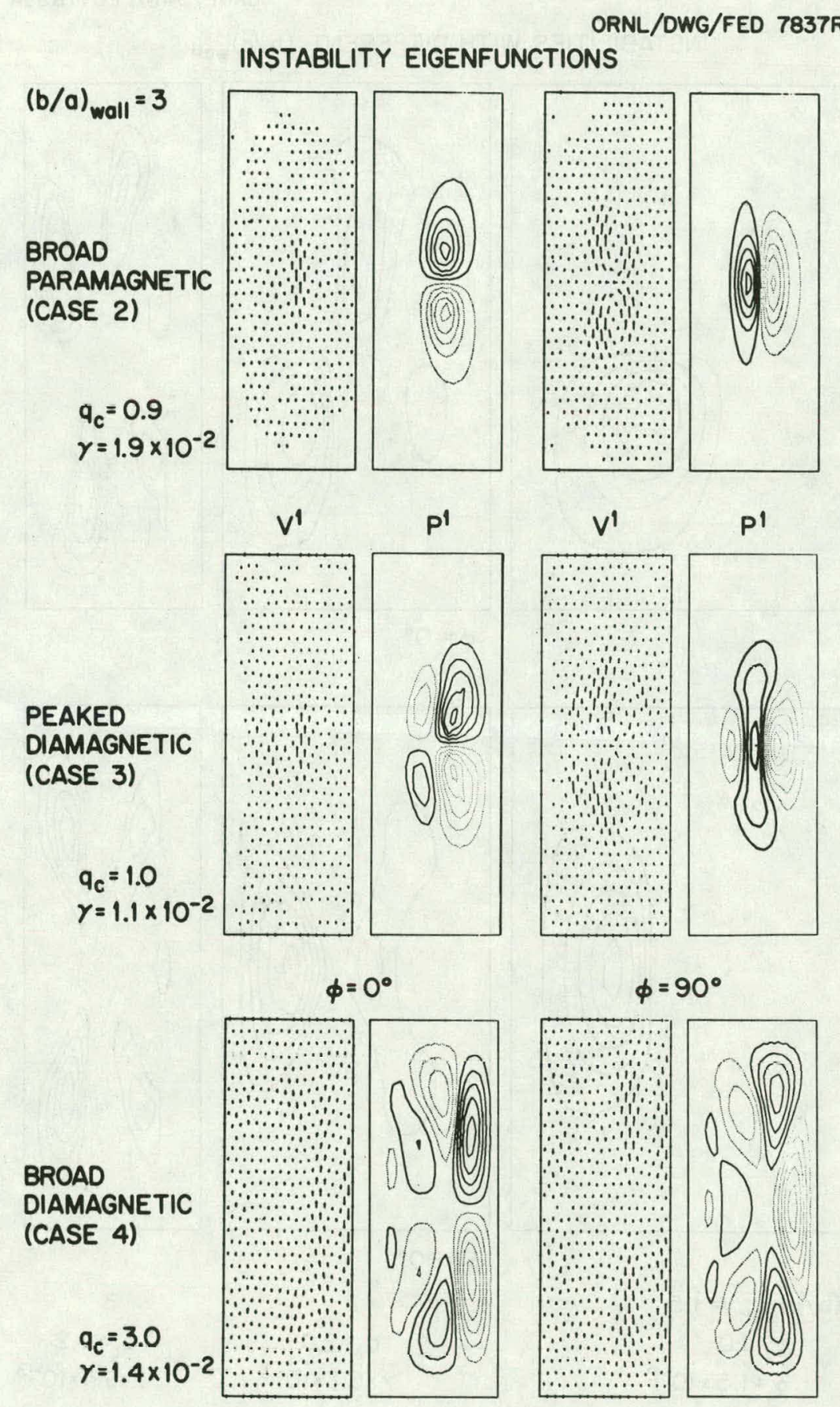

Fig. 9. 
ORNL/DWG/FED-7835R INSTABILITIES WITH DIFFERENT $(b / a)_{\text {wall }}$
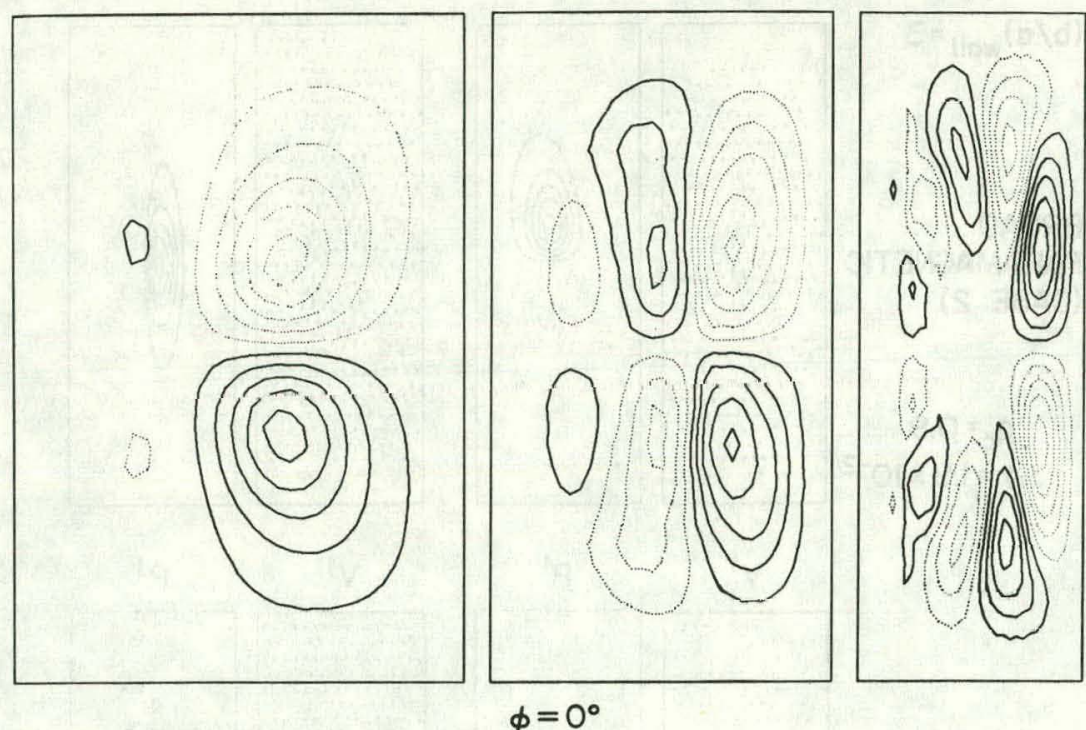

$\phi=0^{\circ}$
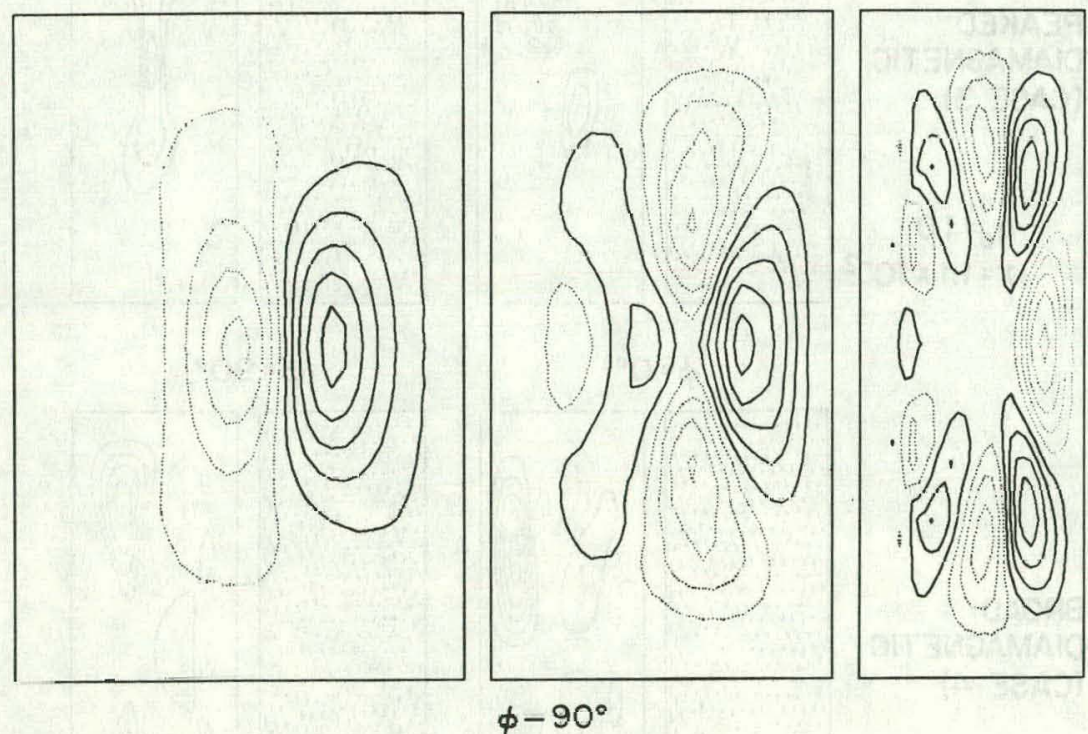

$$
\begin{aligned}
& (b / a)_{\text {wall }}=1.5 \\
& q_{c}=1.0 \\
& y=1.5 \times 10^{-2}
\end{aligned}
$$

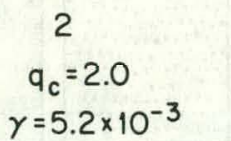

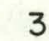

$q_{c}=4.2$ $\gamma=7.4 \times 10^{-3}$

Fig. 10. 
Table I. Cases of equilibria

ASP $=4$
\begin{tabular}{|c|c|c|c|c|c|c|c|c|}
\hline CASE & $N$ & N & A & $\mathrm{C}$ & $\beta_{\text {pol }}$ & $(\mathrm{b} / \mathrm{a})_{\text {axis }} /(\mathrm{b} / \mathrm{a})_{\text {wall }}=3$ & $\Delta / \mathrm{a}$ & CONFIGURATION \\
\hline \hline CASE 1 & 1 & 3 & 1 & 0.3 & 0.83 & 0.66 & 0.099 & PEAKED PARA \\
\hline CASE 2 & 1 & 2 & 1 & 0.3 & 0.81 & 0.92 & 0.093 & BROAD PARA \\
\hline CASE 3 & 1.5 & 3 & 1 & 0.2 & 1.42 & 0.80 & 0.172 & PEAKED DIA \\
\hline CASE 4 & 1 & 3 & 1 & 0.3 & 1.32 & 1.57 & 0.185 & BROAD DIA \\
\hline
\end{tabular}


Table II. Equilibrium conditions near marginal stability

ASP $=4 \quad$ GROWTH RATE $=0.00:$

\begin{tabular}{|c|c|c|c|c|c|}
\hline CASE & $(b / a)_{\text {wall }}$ & $(b / a)_{a x}$ is & $\begin{array}{c}\text { MARGINAL } \\
a_{c}\end{array}$ & $\beta_{F} d_{d}$ & $\beta_{a v}(\%)$ \\
\hline \multirow{2}{*}{$\begin{array}{l}\text { CASE } 1 \\
\text { PEAKED }\end{array}$} & 2 & 1.70 & 0.85 & 1.3? & 1.0 \\
\hline & 3 & 1.98 & 0.88 & 0.33 & 1.2 \\
\hline PARA & 4 & 2.08 & 0.90 & $\mathbf{1 . 3 3}$ & 1.2 \\
\hline \multirow{2}{*}{$\begin{array}{l}\text { CASE } 2 \\
\text { BROAD }\end{array}$} & 2 & 1.98 & 0.95 & C.? & 1.8 \\
\hline & 3 & 2.76 & 1.00 & $C .8 \mathrm{i}$ & 3.0 \\
\hline PARA & 4 & 3.40 . & 1.70 & {$\left[.8_{e}^{+\infty}\right.$} & 1.6 \\
\hline \multirow{2}{*}{$\begin{array}{l}\text { CASE } 3 \\
\text { PEAKED }\end{array}$} & 2 & 1.90 & 0.98 & $1.3 \varepsilon$ & 2.1 \\
\hline & 3 & 2.40 & 1.12 & $\cdot L<\bar{L}$ & 2.1 \\
\hline DIA & 4 & 2.64 & 2.00 & $1.5 C$ & 0.6 \\
\hline CASE 5 & 1.5 & 1.73 & 1.20 & $1 . \bar{c} 0$ & 2.8 \\
\hline BROAD & 2 & 2.44 & 1.90 & 188 & 1.9 \\
\hline$D \mid A$ & 3 & 7.32 & 4.50 & 113 & 1.2 \\
\hline
\end{tabular}


ORNL/TM-6419

Dist. Category UC-20 g

INTERNAL DISTRIBUTION

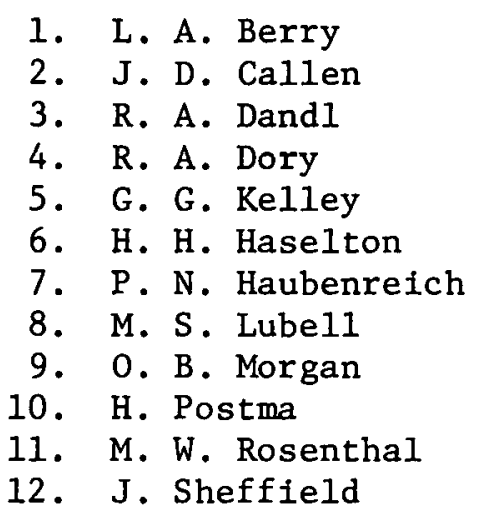

\author{
13. D. Steiner \\ 14-31. C. H. An \\ 32-47. Glenn Bateman \\ 48-49. Laboratory Records Department \\ 50. Laboratory Records, ORNL-RC \\ 51. Document Reference Section \\ 52-53. Central Research Library \\ 54. Fusion Energy Division Library \\ 55. Fusion Energy Division \\ Communications Center \\ 56. ORNL Patent Office
}

\section{EXTERNAL DISTRIBUTION}

57. D. J. Anthony, Energy Systems and Technology Division, General Electric Company, 1 River Road, Bldg. 23, Room 290, Schenectady, NY 12345

58. Bibliothek, Max-Planck Institute für Plasmaphysik, 8046 Garching bei München, Federal Republic of Germany

59. Bibliothèque, Service du Confinement des Plasmas, C.E.A., B.P. No. 6, 92, Fontenay-aux Roses (Seine), France

60. Lung Cheung, Department of Electronics, University Science Center, The Chinese University of Hong Kong, Shatin, N.T., Hong Kong

61. J. F. Clarke, Office of Fusion Energy, G-234, Department of Energy, Washington, DC 20545

62. R. W. Conn, Fusion Technology Program, Nuclear-Engineering Department, University of Wisconsin, Madison, WI 53706

63. CTR Library, c/o Alan F. Haught, United Technologies Research Laboratory, East Hartford, CT 06108

64. CTR Reading Room, c/o Allan N. Kaufman, Physics Department, University of California, Berkeley, CA 94720

65. J. Narl Davidson, School of Nuclear Engineering, Georgia Institute of Technology, Atlanta, GA 30332

66. Documentation S.I.G.N., Départment de la Physique du Plasma et de la Fusion Controlée, Association EURATOM-CEA sur la Fusion, Centre d'Etudes Nucléaires, B.P. 85, Centre du TRI, 38041 Grenoble, Cedex, France

67. W. R. Ellis, Office of Fusion Energy, G-234, Department of Energy, Washington, DC 20545

68. Harold K. Forsen, Exxon Nuclear Co., Inc., 777 106th Avenue, N.E., C-000777, Bellevue, WA 98009

69. Harold P. Furth, Princeton Plasma Physics Laboratory, Princeton University, Forrestal Campus, P. . Box 451, Princeton, NJ 08540

70. Rny W. Gould, California Institute of Technology, Mail Stop 116-81, Pasadena, CA 91125 
71. Robert L. Hirsch, Exxon Research and Engineering, P.0. Box 101, Florham Park, NJ 07932

72. Raymond A. Huse, Manager, Research and Development, Public Service Gas and Electric Company, 80 Park Place, Newark, NJ 07101

73. T. Hsu, Office of Fusion Energy, G-234, Department of Energy, Washington, DC 20545

74. V. E. Ivanov, Physica1-Technical Institute of the Ilkranian Academy of Sciences, Sukhumi, U.S.S.R.

75. A. Kadish, Office of Fusion Energy, G-234, Department of Energy, Washington, DC 20545

76. L. M. Kovrizhnikh, Lebedev Institute of Physics, Academy of Sciences of the U.S.S.R,, Leninsky Prnspect 53, Moecow, U.S.S.R.

77. Guy Laval, Groupe de Physique Théorique, Ecole Polytechnique, 91. Palaispau, Parie, Francc

78. Library, Centre de Recherches en Physique des Plasma, 21 Avenue des Bains, 1007, Lausanne, Switzerland

79. Library, Culham Laboratory, United Kingdom Atomic Energy Authority, Abingdon, Oxon, OX14 3DB, United Kingdom

80. Library, FOM-Institut voor Plasma - Fysica, Rijnhuizen, Jutphaas, Netherlands

81. Library, Institute for Plasma Physics, Nagnya University, Nagoya, Japan 464

82. Library, International Centre for Theoretical Physics, Trieste, Italy

83. Library, Laboratorio Gas Ionizzati, Frascati, Italy

84. Dsumber G. Lominadze, Academy of Sciences of the Genrgian S.S.R., 8 Dzerzhinski St., 38004, Thi1isi, II.S.S.R.

85. Oscar P. Manley, Office of Fusion Energy, G-234, Department of Energy, Washington, DC 20545

86. D. G. McNlcco, Exxon Nucledil Cu., Ine., Reseàrch and Technology Laser Eniilicluent Department, zybs George Washington Way, Kichland, WA 99352

87. J. E. McCune, School of Engineering, Department of Aeronautics and Astronautics, Bldg. 37-391, Masssachusetts Tnstitute of Technology, Cambridge, MA 02139

88. Claude Mercier, Service du Theorie des Plasmas, Centre d'Études Nucléaires, Fontenay-aux-Roses (Seine), France

8y. K. G. Moses, Office of Fusion Energy, G-234, Department of Energy, Washington, DC 20545

90. D. Pfirsch, Inst1tute for Plasma Physics, 8046 Garching bej. Minchen, Federal Republic of Germany

91. Plasma Physics Group, Department of Engineering Physics, Australian National University, P.O. Box 4, Canberra A.C.T. 2600, Australia

92. A. Rogister, Institute for Plasma Physics, KFA, Postfach 1913, D-5170, Jultch 1, Federal Republic of Germany

93. W. Sadowski, Office of Fusion Energy, G-234, Department of Energy, Washington, DC 20545

94. V. D. Shafranov, I. V. Kurchatov Institute of Atomic Energy, 46 Ulitsa Kurchatova, P.0. Box 3402, Moscow, U.S.S.R. 
95. Yu. S. Sigov, Institute of Applied Mathematics of the U.S.S.R. Academy of Sciences, Miuskaya, Sq. 4, Moscow A-47, U.S.S.R.

96. W. M. Stacey, Jr., School of Nuclear Engineering, Georgia Institute of Technology, Atlanta, GA 30332

97. J. B. Taylor, Culham Laboratory, United Kingdom Atomic Energy Author1ty, Abingdon, Oxon, Ox14 3DB, Unfted Kingdom

98. Thermonuclear Library, Japan Atomic Energy Research Institute, Toka1, Naka, Ibaraki, Japan

99. Francisco Verdaguer, Director, Division of Fusion, Junta de Energia Nuclear, Madrid 3, Spain

100. Director, Research and Technical Support Division, Department of Energy, Oak Ridge Operations, P.O. Box E, Oak Ridge, TN 37830

101-276. Given distribution as shown in TID-4500, Magnetic Fusion Energy (Distribution Category UC-20 g, Theoretical Plasma Physics) 